2 Source attribution of black carbon and its direct radiative forcing

3

4

5

6

\section{in China}

Yang Yang ${ }^{1}$, Hailong Wang ${ }^{1 *}$, Steven J. Smith ${ }^{2}$, Po-Lun Ma ${ }^{1}$, Philip J. Rasch ${ }^{1}$

${ }^{1}$ Atmospheric Science and Global Change Division, Pacific Northwest National Laboratory, Richland, Washington, USA

${ }^{2}$ Joint Global Change Research Institute, Pacific Northwest National Laboratory, College Park, Maryland, USA

*Correspondence to yang.yang@pnnl.gov and hailong.wang@pnnl.gov 
19 Table S1. Comparisons of observed and modeled seasonal mean near-surface 20 concentrations (units: $\mathrm{g} \mathrm{m} \mathrm{m}^{-3}$ ) of BC in China corresponding to Fig. 3a. Numbers in 21 bold represent sites with observed concentration lower than modeled concentration, 22 otherwise the observation higher than modeled concentration.

23

\begin{tabular}{|c|c|c|c|c|c|c|c|c|c|}
\hline & \multirow[b]{2}{*}{ Sites } & \multicolumn{2}{|c|}{ DJF } & \multicolumn{2}{|c|}{ MAM } & \multicolumn{2}{|c|}{ JJA } & \multicolumn{2}{|c|}{ SON } \\
\hline & & Obs. & Model & Obs. & Model & Obs. & Model & Obs. & Model \\
\hline \multirow{3}{*}{$\mathrm{NC}$} & Gucheng & $1.687 \mathrm{E}+01$ & $6.332 \mathrm{E}+00$ & $6.939 \mathrm{E}+00$ & $3.597 \mathrm{E}+00$ & $7.038 \mathrm{E}+00$ & $3.089 \mathrm{E}+00$ & $1.155 \mathrm{E}+01$ & $4.606 \mathrm{E}+00$ \\
\hline & Linan & $4.831 E+00$ & $5.830 \mathrm{E}+00$ & 4.167E+00 & $2.629 \mathrm{E}+00$ & $3.735 \mathrm{E}+00$ & $1.993 \mathrm{E}+00$ & $4.133 E+00$ & $2.829 \mathrm{E}+00$ \\
\hline & Zhengzhou & $1.270 \mathrm{E}+01$ & $9.171 \mathrm{E}+00$ & $8.034 \mathrm{E}+00$ & $3.974 \mathrm{E}+00$ & $6.939 \mathrm{E}+00$ & $3.404 \mathrm{E}+00$ & $9.894 \mathrm{E}+00$ & $5.173 \mathrm{E}+00$ \\
\hline \multirow{3}{*}{ SC } & Jinsha & $3.436 \mathrm{E}+00$ & $6.708 \mathrm{E}+00$ & $2.241 \mathrm{E}+00$ & $3.016 \mathrm{E}+00$ & $1.892 \mathrm{E}+00$ & $2.252 \mathrm{E}+00$ & $4.283 E+00$ & $3.446 \mathrm{E}+00$ \\
\hline & Panyu & $9.628 \mathrm{E}+00$ & $5.080 \mathrm{E}+00$ & $8.134 \mathrm{E}+00$ & $2.928 \mathrm{E}+00$ & $4.847 \mathrm{E}+00$ & $1.864 \mathrm{E}+00$ & 7.437E+00 & $3.646 \mathrm{E}+00$ \\
\hline & Taiyangshan & $2.623 E+00$ & $7.346 \mathrm{E}+00$ & $2.042 \mathrm{E}+00$ & $3.410 \mathrm{E}+00$ & $2.092 \mathrm{E}+00$ & $2.408 \mathrm{E}+00$ & $3.652 \mathrm{E}+00$ & $4.038 \mathrm{E}+00$ \\
\hline \multirow{2}{*}{ SW } & Chengdu & $1.147 \mathrm{E}+01$ & $6.828 \mathrm{E}+00$ & $1.072 \mathrm{E}+01$ & $2.831 \mathrm{E}+00$ & $9.728 \mathrm{E}+00$ & $2.509 E+00$ & $1.106 \mathrm{E}+01$ & $3.617 \mathrm{E}+00$ \\
\hline & Nanning & $4.980 \mathrm{E}+00$ & $3.074 \mathrm{E}+00$ & $2.623 E+00$ & $1.579 \mathrm{E}+00$ & $2.722 \mathrm{E}+00$ & $8.660 \mathrm{E}-01$ & $4.980 \mathrm{E}+00$ & $1.926 \mathrm{E}+00$ \\
\hline \multirow{2}{*}{$\mathrm{CW}$} & Gaolanshan & $5.279 \mathrm{E}+00$ & $2.211 \mathrm{E}+00$ & $2.789 \mathrm{E}+00$ & $9.690 \mathrm{E}-01$ & $2.872 \mathrm{E}+00$ & $9.000 \mathrm{E}-01$ & $4.050 \mathrm{E}+00$ & $1.302 \mathrm{E}+00$ \\
\hline & Xian & $1.853 \mathrm{E}+01$ & $6.465 E+00$ & $1.145 \mathrm{E}+01$ & $2.664 \mathrm{E}+00$ & $7.570 \mathrm{E}+00$ & $2.173 \mathrm{E}+00$ & $1.077 \mathrm{E}+01$ & $3.360 \mathrm{E}+00$ \\
\hline \multirow{2}{*}{$\mathrm{NE}$} & Dalian & $7.520 \mathrm{E}+00$ & $2.093 E+00$ & $4.548 \mathrm{E}+00$ & $1.525 \mathrm{E}+00$ & $3.519 \mathrm{E}+00$ & $9.770 \mathrm{E}-01$ & $5.428 \mathrm{E}+00$ & $1.277 \mathrm{E}+00$ \\
\hline & TYS & $3.818 \mathrm{E}+00$ & $2.636 \mathrm{E}+00$ & $1.527 \mathrm{E}+00$ & $1.440 \mathrm{E}+00$ & $1.112 \mathrm{E}+00$ & $9.790 \mathrm{E}-01$ & $2.507 \mathrm{E}+00$ & $1.698 \mathrm{E}+00$ \\
\hline NW & Dunhuang & $5.760 \mathrm{E}+00$ & $2.090 \mathrm{E}-01$ & $2.556 \mathrm{E}+00$ & $1.340 \mathrm{E}-01$ & $3.436 \mathrm{E}+00$ & $1.220 \mathrm{E}-01$ & $4.548 E+00$ & $1.550 \mathrm{E}-01$ \\
\hline TP & Lhasa & $5.428 \mathrm{E}+00$ & $1.840 \mathrm{E}-01$ & $3.021 \mathrm{E}+00$ & $2.420 \mathrm{E}-01$ & $3.469 \mathrm{E}+00$ & $7.100 \mathrm{E}-02$ & $3.486 E+00$ & $1.300 \mathrm{E}-01$ \\
\hline
\end{tabular}
24

25

26 
27 Table S2. Comparisons of observed and modeled seasonal mean aerosol absorption 28 optical depth (AAOD) of BC in China corresponding to Fig. 3b. Numbers in bold 29 represent sites with observed concentration lower than modeled concentration, 30 otherwise the observation higher than modeled concentration. Lack data show in 31 blank.

32

\begin{tabular}{|c|c|c|c|c|c|c|c|c|c|}
\hline & \multirow[b]{2}{*}{ Sites } & \multicolumn{2}{|c|}{ DJF } & \multicolumn{2}{|c|}{ MAM } & \multicolumn{2}{|c|}{ JJA } & \multicolumn{2}{|c|}{ SON } \\
\hline & & Obs. & Model & Obs. & Model & Obs. & Model & Obs. & Model \\
\hline \multirow{6}{*}{$\mathrm{NC}$} & Beijing & $6.430 \mathrm{E}-02$ & $2.986 \mathrm{E}-02$ & 5.568E-02 & 3.699E-02 & 4.286E-02 & 4.182E-02 & 5.303E-02 & 3.361E-02 \\
\hline & Xianghe & 6.658E-02 & 2.986E-02 & 5.646E-02 & 3.699E-02 & 3.480E-02 & 4.182E-02 & $6.412 \mathrm{E}-02$ & 3.361E-02 \\
\hline & Xinglong & 3.535E-02 & 2.986E-02 & 3.249E-02 & 3.699E-02 & 2.612E-02 & 4.182E-02 & 2.531E-02 & 3.361E-02 \\
\hline & Taihu & 4.684E-02 & 6.860E-02 & 4.122E-02 & 4.683E-02 & 3.581E-02 & 3.754E-02 & 4.073E-02 & 4.020E-02 \\
\hline & Hefei & 6.600E-02 & 7.464E-02 & 3.700E-02 & 4.926E-02 & & & 4.050E-02 & 4.406E-02 \\
\hline & Shouxian & $6.400 \mathrm{E}-02$ & 8.011E-02 & 2.700E-02 & 5.215E-02 & $2.300 \mathrm{E}-02$ & 4.263E-02 & 4.967E-02 & 4.844E-02 \\
\hline \multirow{3}{*}{ SC } & Chen-Kung U. & 1.468E-02 & 2.437E-02 & 1.903E-02 & 1.835E-02 & 7.186E-03 & 8.903E-03 & 8.059E-03 & 1.098E-02 \\
\hline & Polytechnic U. & 3.642E-02 & $2.735 \mathrm{E}-02$ & 3.790E-02 & 1.987E-02 & 5.125E-02 & 9.388E-03 & 3.863E-02 & 1.637E-02 \\
\hline & Hok Tsui & 3.733E-02 & $2.735 \mathrm{E}-02$ & 4.733E-02 & 1.987E-02 & 4.500E-02 & 9.388E-03 & $2.500 \mathrm{E}-02$ & 1.637E-02 \\
\hline $\mathrm{CW}$ & SACOL & 3.047E-02 & 1.925E-02 & 3.171E-02 & 1.794E-02 & 2.459E-02 & 1.950E-02 & 2.288E-02 & 1.828E-02 \\
\hline
\end{tabular}


33 Table S3. Contributions from tagged source regions (S, column) to regional mean 34 surface concentrations of $\mathrm{BC}\left(\mathrm{g} \mathrm{m}^{-3}\right)$ over the seven receptor regions in China and 35 China ( $R$, row) in December-January-February (DJF), March-April-May (MAM), 36 June-July-August (JJA), and September-October-November (SON).

\begin{tabular}{|c|c|c|c|c|c|c|c|c|}
\hline \multicolumn{9}{|c|}{ DJF } \\
\hline$S \quad R$ & $\mathrm{NC}$ & $\mathrm{SC}$ & SW & $\mathrm{CW}$ & NE & NW & TP & $\mathrm{CN}$ \\
\hline $\mathrm{NC}$ & $5.793 E+00$ & $1.289 \mathrm{E}+00$ & $5.014 \mathrm{E}-01$ & $2.135 \mathrm{E}-01$ & $1.453 \mathrm{E}-01$ & 6.057E-04 & $8.484 \mathrm{E}-03$ & $1.115 \mathrm{E}+00$ \\
\hline SC & 1.497E-01 & $2.740 \mathrm{E}+00$ & 6.591E-01 & $5.026 \mathrm{E}-02$ & $5.030 \mathrm{E}-04$ & 1.717E-04 & $1.391 \mathrm{E}-02$ & $4.001 \mathrm{E}-01$ \\
\hline SW & 6.907E-02 & 7.979E-02 & $2.106 \mathrm{E}+00$ & 2.189E-01 & $1.014 \mathrm{E}-03$ & $3.279 \mathrm{E}-04$ & $2.224 \mathrm{E}-02$ & $2.359 \mathrm{E}-01$ \\
\hline $\mathrm{CW}$ & 1.247E-01 & $5.615 \mathrm{E}-02$ & 8.148E-02 & $1.028 \mathrm{E}+00$ & 7.969E-03 & $2.548 \mathrm{E}-03$ & $1.905 \mathrm{E}-03$ & 1.184E-01 \\
\hline NE & 5.103E-02 & $7.271 \mathrm{E}-03$ & $1.610 \mathrm{E}-03$ & 3.652E-04 & $1.020 \mathrm{E}+00$ & $1.324 \mathrm{E}-05$ & 3.031E-05 & $1.539 \mathrm{E}-01$ \\
\hline NW & $1.570 \mathrm{E}-02$ & 7.569E-03 & 5.851E-03 & 3.849E-02 & $5.227 \mathrm{E}-03$ & 2.967E-01 & $5.280 \mathrm{E}-04$ & 7.559E-02 \\
\hline TP & 2.647E-03 & $5.986 \mathrm{E}-03$ & 3.483E-02 & $6.624 \mathrm{E}-03$ & $6.045 \mathrm{E}-05$ & 2.153E-03 & 8.348E-02 & $2.121 \mathrm{E}-02$ \\
\hline RW & 4.759E-02 & $1.229 \mathrm{E}-01$ & $1.641 \mathrm{E}-01$ & 4.306E-02 & 2.991E-02 & 8.403E-02 & 1.737E-01 & $9.626 \mathrm{E}-02$ \\
\hline \multicolumn{9}{|c|}{ MAM } \\
\hline $\mathrm{NC}$ & $2.631 \mathrm{E}+00$ & 2.837E-01 & $1.058 \mathrm{E}-01$ & 1.154E-01 & $1.930 \mathrm{E}-01$ & $1.100 \mathrm{E}-03$ & 2.014E-03 & 4.804E-01 \\
\hline SC & $1.556 \mathrm{E}-01$ & $1.614 \mathrm{E}+00$ & 2.161E-01 & $2.255 \mathrm{E}-02$ & 4.397E-03 & $3.414 \mathrm{E}-04$ & 3.331E-03 & 2.292E-01 \\
\hline SW & 3.816E-02 & 3.322E-02 & $9.988 \mathrm{E}-01$ & $1.345 \mathrm{E}-01$ & 3.407E-03 & $5.673 \mathrm{E}-04$ & 4.062E-03 & $1.139 \mathrm{E}-01$ \\
\hline $\mathrm{CW}$ & $2.990 \mathrm{E}-02$ & $6.613 \mathrm{E}-03$ & $1.579 \mathrm{E}-02$ & 3.894E-01 & 7.070E-03 & $3.688 \mathrm{E}-03$ & $6.514 \mathrm{E}-04$ & 4.036E-02 \\
\hline NE & 3.649E-02 & $7.578 \mathrm{E}-03$ & $1.230 \mathrm{E}-03$ & 1.495E-03 & $5.124 \mathrm{E}-01$ & 1.122E-04 & 2.338E-05 & 7.963E-02 \\
\hline NW & 3.874E-03 & $1.469 \mathrm{E}-03$ & $1.488 \mathrm{E}-03$ & $1.373 \mathrm{E}-02$ & $2.766 \mathrm{E}-03$ & 9.793E-02 & $1.329 \mathrm{E}-03$ & $2.505 \mathrm{E}-02$ \\
\hline TP & $1.995 \mathrm{E}-03$ & $4.814 \mathrm{E}-03$ & $2.748 \mathrm{E}-02$ & $2.616 \mathrm{E}-03$ & $1.380 \mathrm{E}-04$ & $4.511 \mathrm{E}-04$ & $5.312 \mathrm{E}-02$ & 1.382E-02 \\
\hline RW & $9.188 \mathrm{E}-02$ & $1.884 \mathrm{E}-01$ & $2.730 \mathrm{E}-01$ & $4.523 \mathrm{E}-02$ & $6.837 \mathrm{E}-02$ & 7.014E-02 & $2.054 \mathrm{E}-01$ & $1.290 \mathrm{E}-01$ \\
\hline \multicolumn{9}{|c|}{ JJA } \\
\hline NC & $2.004 \mathrm{E}+00$ & 4.844E-02 & 2.846E-02 & 1.215E-01 & $2.503 \mathrm{E}-01$ & 3.223E-03 & $5.409 \mathrm{E}-04$ & $3.600 \mathrm{E}-01$ \\
\hline SC & $2.736 \mathrm{E}-01$ & $1.234 \mathrm{E}+00$ & $1.108 \mathrm{E}-01$ & 5.379E-02 & 2.797E-02 & $1.112 \mathrm{E}-03$ & $2.229 \mathrm{E}-03$ & $2.004 \mathrm{E}-01$ \\
\hline SW & 2.692E-02 & $1.967 \mathrm{E}-02$ & 8.639E-01 & 2.194E-01 & $6.650 \mathrm{E}-03$ & $5.495 \mathrm{E}-03$ & 8.326E-03 & $1.075 \mathrm{E}-01$ \\
\hline $\mathrm{CW}$ & $1.022 \mathrm{E}-02$ & $2.836 \mathrm{E}-04$ & 4.260E-03 & 2.769E-01 & 4.605E-03 & $9.085 \mathrm{E}-03$ & $1.659 \mathrm{E}-03$ & $2.752 \mathrm{E}-02$ \\
\hline NE & $1.035 \mathrm{E}-02$ & $2.630 \mathrm{E}-04$ & $6.470 \mathrm{E}-05$ & $9.615 \mathrm{E}-04$ & $3.480 \mathrm{E}-01$ & $1.288 \mathrm{E}-04$ & 1.354E-05 & $5.125 \mathrm{E}-02$ \\
\hline NW & $1.001 \mathrm{E}-03$ & 1.649E-04 & 5.722E-04 & 6.316E-03 & $1.150 \mathrm{E}-03$ & 7.543E-02 & $5.272 \mathrm{E}-03$ & $1.915 \mathrm{E}-02$ \\
\hline TP & 7.957E-04 & $8.475 \mathrm{E}-04$ & 1.749E-02 & $2.431 \mathrm{E}-03$ & $1.415 \mathrm{E}-04$ & $2.908 \mathrm{E}-04$ & 3.105E-02 & $8.020 \mathrm{E}-03$ \\
\hline RW & 8.071E-02 & 1.210E-01 & 7.176E-02 & 2.047E-02 & $6.054 \mathrm{E}-02$ & 4.822E-02 & 4.372E-02 & $6.221 \mathrm{E}-02$ \\
\hline \multicolumn{9}{|c|}{ SON } \\
\hline $\mathrm{NC}$ & $3.239 E+00$ & $4.962 \mathrm{E}-01$ & $2.577 \mathrm{E}-01$ & $1.680 \mathrm{E}-01$ & $2.060 \mathrm{E}-01$ & $1.405 \mathrm{E}-03$ & 7.070E-03 & $6.182 \mathrm{E}-01$ \\
\hline SC & $1.300 \mathrm{E}-01$ & $1.739 \mathrm{E}+00$ & 3.896E-01 & 4.141E-02 & $2.950 \mathrm{E}-03$ & $2.873 \mathrm{E}-04$ & $1.014 \mathrm{E}-02$ & $2.580 \mathrm{E}-01$ \\
\hline SW & 3.083E-02 & 1.919E-02 & $1.217 \mathrm{E}+00$ & $1.826 \mathrm{E}-01$ & $3.085 \mathrm{E}-03$ & 1.156E-03 & 2.309E-02 & $1.390 \mathrm{E}-01$ \\
\hline $\mathrm{CW}$ & 4.234E-02 & $1.015 \mathrm{E}-02$ & 2.033E-02 & $5.113 \mathrm{E}-01$ & $1.035 \mathrm{E}-02$ & 4.947E-03 & $8.765 \mathrm{E}-04$ & 5.377E-02 \\
\hline $\mathrm{NE}$ & 4.230E-02 & $1.230 \mathrm{E}-02$ & 4.253E-03 & $1.685 \mathrm{E}-03$ & $6.537 \mathrm{E}-01$ & $6.916 \mathrm{E}-05$ & $1.502 \mathrm{E}-04$ & $1.015 \mathrm{E}-01$ \\
\hline NW & 6.967E-03 & $2.650 \mathrm{E}-03$ & $2.562 \mathrm{E}-03$ & $2.018 \mathrm{E}-02$ & $4.738 \mathrm{E}-03$ & $1.549 \mathrm{E}-01$ & $1.782 \mathrm{E}-03$ & $3.968 \mathrm{E}-02$ \\
\hline TP & $1.139 \mathrm{E}-03$ & 1.127E-03 & $1.748 \mathrm{E}-02$ & $3.623 \mathrm{E}-03$ & $9.359 \mathrm{E}-05$ & $5.573 \mathrm{E}-04$ & 6.368E-02 & $1.446 \mathrm{E}-02$ \\
\hline RW & 6.893E-02 & $1.185 \mathrm{E}-01$ & $5.844 \mathrm{E}-02$ & $2.435 \mathrm{E}-02$ & 3.891E-02 & 6.036E-02 & $8.202 \mathrm{E}-02$ & 6.620E-02 \\
\hline
\end{tabular}


39 Table S4. Seasonal and annual direct radiative effect (DRF) efficiency of BC ( $\left.\mathrm{W} \mathrm{m}^{-2}\right)$ 40 for each of the tagged source regions over China and globally, respectively. The 41 efficiency is defined as the DRF divided by the corresponding scaled annual emission 42 (seasonal emission multiplied by 4).

43

\begin{tabular}{lccccc}
\hline China & DJF & MAM & JJA & SON & ANN \\
\hline NC & $3.571 \mathrm{E}-01$ & $5.491 \mathrm{E}-01$ & $7.189 \mathrm{E}-01$ & $5.204 \mathrm{E}-01$ & $5.126 \mathrm{E}-01$ \\
SC & $3.644 \mathrm{E}-01$ & $5.563 \mathrm{E}-01$ & $7.596 \mathrm{E}-01$ & $5.928 \mathrm{E}-01$ & $5.574 \mathrm{E}-01$ \\
SW & $6.950 \mathrm{E}-01$ & $8.289 \mathrm{E}-01$ & $8.660 \mathrm{E}-01$ & $7.377 \mathrm{E}-01$ & $7.714 \mathrm{E}-01$ \\
CW & $6.433 \mathrm{E}-01$ & $9.416 \mathrm{E}-01$ & $1.106 \mathrm{E}+00$ & $6.780 \mathrm{E}-01$ & $8.034 \mathrm{E}-01$ \\
NE & $2.562 \mathrm{E}-01$ & $3.530 \mathrm{E}-01$ & $4.673 \mathrm{E}-01$ & $2.453 \mathrm{E}-01$ & $3.154 \mathrm{E}-01$ \\
NW & $9.674 \mathrm{E}-01$ & $1.652 \mathrm{E}+00$ & $3.174 \mathrm{E}+00$ & $1.556 \mathrm{E}+00$ & $1.670 \mathrm{E}+00$ \\
TP & $9.499 \mathrm{E}-01$ & $1.182 \mathrm{E}+00$ & $9.443 \mathrm{E}-01$ & $9.421 \mathrm{E}-01$ & $1.015 \mathrm{E}+00$ \\
\hline Global & DJF & MAM & JJA & SON & ANN \\
\hline NC & $1.701 \mathrm{E}-02$ & $4.056 \mathrm{E}-02$ & $3.537 \mathrm{E}-02$ & $2.253 \mathrm{E}-02$ & $2.741 \mathrm{E}-02$ \\
SC & $2.081 \mathrm{E}-02$ & $3.241 \mathrm{E}-02$ & $3.080 \mathrm{E}-02$ & $2.878 \mathrm{E}-02$ & $2.784 \mathrm{E}-02$ \\
SW & $3.436 \mathrm{E}-02$ & $4.387 \mathrm{E}-02$ & $3.434 \mathrm{E}-02$ & $3.493 \mathrm{E}-02$ & $3.672 \mathrm{E}-02$ \\
CW & $2.492 \mathrm{E}-02$ & $5.780 \mathrm{E}-02$ & $5.070 \mathrm{E}-02$ & $2.758 \mathrm{E}-02$ & $3.778 \mathrm{E}-02$ \\
NE & $1.078 \mathrm{E}-02$ & $4.483 \mathrm{E}-02$ & $3.562 \mathrm{E}-02$ & $1.790 \mathrm{E}-02$ & $2.575 \mathrm{E}-02$ \\
NW & $2.863 \mathrm{E}-02$ & $9.540 \mathrm{E}-02$ & $1.567 \mathrm{E}-01$ & $6.668 \mathrm{E}-02$ & $7.661 \mathrm{E}-02$ \\
TP & $4.681 \mathrm{E}-02$ & $7.488 \mathrm{E}-02$ & $4.435 \mathrm{E}-02$ & $4.937 \mathrm{E}-02$ & $5.564 \mathrm{E}-02$ \\
RW & $1.701 \mathrm{E}-02$ & $4.056 \mathrm{E}-02$ & $3.537 \mathrm{E}-02$ & $2.253 \mathrm{E}-02$ & $2.741 \mathrm{E}-02$ \\
\hline
\end{tabular}

44 
Table S5. Seasonal and annual near-surface concentration $\left(\mu \mathrm{g} \mathrm{m}^{-3}\right)$ and column burden $\left(\mathrm{mg} \mathrm{m}^{-2}\right)$ efficiency of BC for each of the tagged source regions over China 48 and globally, respectively.

\begin{tabular}{|c|c|c|c|c|c|}
\hline & \multicolumn{5}{|c|}{ Near-Surface Concentration Efficiency } \\
\hline China & DJF & MAM & JJA & SON & ANN \\
\hline NC & 7.457E-01 & 4.917E-01 & $3.961 \mathrm{E}-01$ & 6.345E-01 & 922E-01 \\
\hline SC & 989E-01 & $.096 \mathrm{E}-01$ & $3.605 \mathrm{E}-01$ & $.701 \mathrm{E}-01$ & 4.652E-01 \\
\hline SW & 10E-01 & $3.000 \mathrm{E}-01$ & 2.894E-01 & 3.727E-01 & $3.726 \mathrm{E}-01$ \\
\hline $\mathrm{CW}$ & 485E-01 & 4.036E-01 & $3.256 \mathrm{E}-01$ & $5.108 \mathrm{E}-01$ & 5.376E-01 \\
\hline IE & 312E-01 & 3.683E-01 & 3.522E-01 & $4.828 \mathrm{E}-01$ & 4.603E-01 \\
\hline IW & 15E-01 & 4.918E-01 & 4.702E-01 & 7.397E-01 & 7.109E-01 \\
\hline TP & 009E-01 & 2.266E-01 & 1.967E-01 & 3.307E-01 & 2.898E-01 \\
\hline Glob & DJF & MAM & JJA & SON & ANN \\
\hline NC & 876E-02 & 1.229E-02 & 8.863E-03 & $1.502 \mathrm{E}-02$ & 1.445E-02 \\
\hline $\mathrm{SC}$ & 88E-02 & 1.006E-02 & 7.981E-03 & $1.281 \mathrm{E}-02$ & 1.213E-02 \\
\hline SW & 24E-02 & $6.939 \mathrm{E}-03$ & 6.081E-03 & $9.305 \mathrm{E}-03$ & $9.129 \mathrm{E}-03$ \\
\hline SW & $5 \mathrm{E}-02$ & $9.510 \mathrm{E}-03$ & 7.178E-03 & 1.149E-02 & $1.247 \mathrm{E}-02$ \\
\hline$E$ & $9 \mathrm{E}-02$ & 1.191E-02 & $9.091 \mathrm{E}-03$ & 1.396E-02 & 1.316E-02 \\
\hline W & 8E-02 & 1.337E-02 & 1.103E-02 & $1.872 \mathrm{E}-02$ & 1.822E-02 \\
\hline TP & 2E-02 & 6.117E-03 & 4.477E-03 & 8.917E-03 & 8.031E-03 \\
\hline \multirow[t]{2}{*}{ RW } & 0E-02 & 1.226E-02 & 1.213E-02 & 1.346E-02 & 1.317E-02 \\
\hline & \multicolumn{5}{|c|}{ Column Burden Efficiency } \\
\hline China & DJF & MAM & JJA & SON & ANN \\
\hline $\mathrm{NC}$ & 460E-01 & 4.588E-01 & 4.007E-01 & 5.345E-01 & $4.948 \mathrm{E}-01$ \\
\hline SC & 960 E- 01 & 4.099E-01 & $4.665 \mathrm{E}-01$ & $4.726 \mathrm{E}-01$ & 4.623E-01 \\
\hline SW & 927E-01 & 3.825E-01 & $3.865 \mathrm{E}-01$ & $4.421 \mathrm{E}-01$ & $4.575 \mathrm{E}-01$ \\
\hline CW & 089E-01 & $5.172 \mathrm{E}-01$ & 4.507E-01 & $5.175 \mathrm{E}-01$ & 5.729E-01 \\
\hline NE & 477E-01 & 2.665E-01 & $2.736 \mathrm{E}-01$ & 2.906E-01 & $2.676 \mathrm{E}-01$ \\
\hline NW & 982E-01 & 6.861E-01 & $1.023 E+00$ & 8.382E-01 & 7.527E-01 \\
\hline TP & $.506 \mathrm{E}-01$ & 4.055E-01 & 3.213E-01 & 4.270E-01 & 4.312E-01 \\
\hline Glob: & DJF & MAM & JJA & SON & ANN \\
\hline $\mathrm{IC}$ & $375 \mathrm{E}-02$ & $1.865 \mathrm{E}-02$ & $1.308 \mathrm{E}-02$ & 1.750E-02 & $1.732 \mathrm{E}-02$ \\
\hline SC & 35E-02 & $1.548 \mathrm{E}-02$ & $1.390 \mathrm{E}-02$ & 1.814E-02 & 1.682E-02 \\
\hline SV & 288E-02 & 1.533E-02 & 1.163E-02 & 1.708E-02 & 1.710E-02 \\
\hline CI & 13E-02 & 2.143E-02 & 1.585E-02 & 1.690E-02 & 1.992E-02 \\
\hline NE & 29E-02 & 1.905E-02 & 1.313E-02 & 1.555E-02 & 1.473E-02 \\
\hline NW & 25E-02 & 3.080E-02 & 4.100E-02 & 3.187E-02 & 2.892E-02 \\
\hline 15 & 713E-02 & 2.282E-02 & 1.393E-02 & 2.051E-02 & $2.166 \mathrm{E}-02$ \\
\hline RW & 2.509E-02 & 2.609E-02 & 2.681E-02 & $2.722 \mathrm{E}-02$ & 2.633E-02 \\
\hline
\end{tabular}




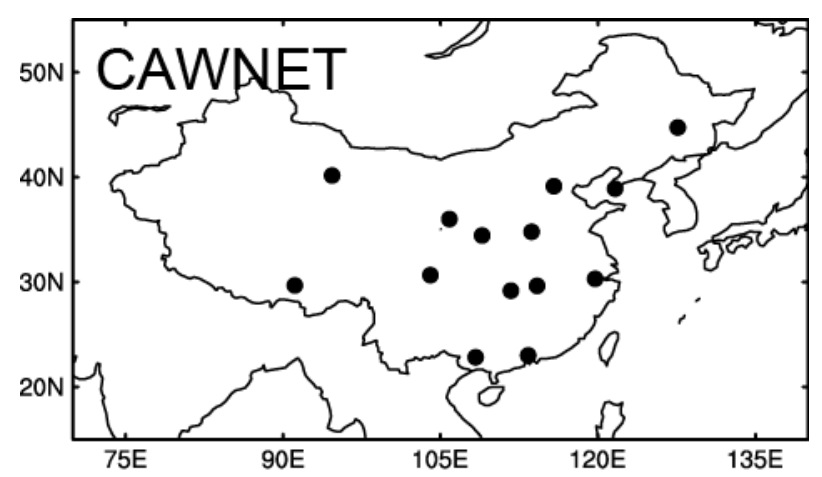

Figure S1. Locations of 14 sites of the China Meteorological Administration (CMA) Atmosphere Watch Network (CAWNET, top) (Zhang et al., 2012) and 10 sites of the Aerosol Robotic Network (AERONET, bottom) (Holben et al., 2001). 

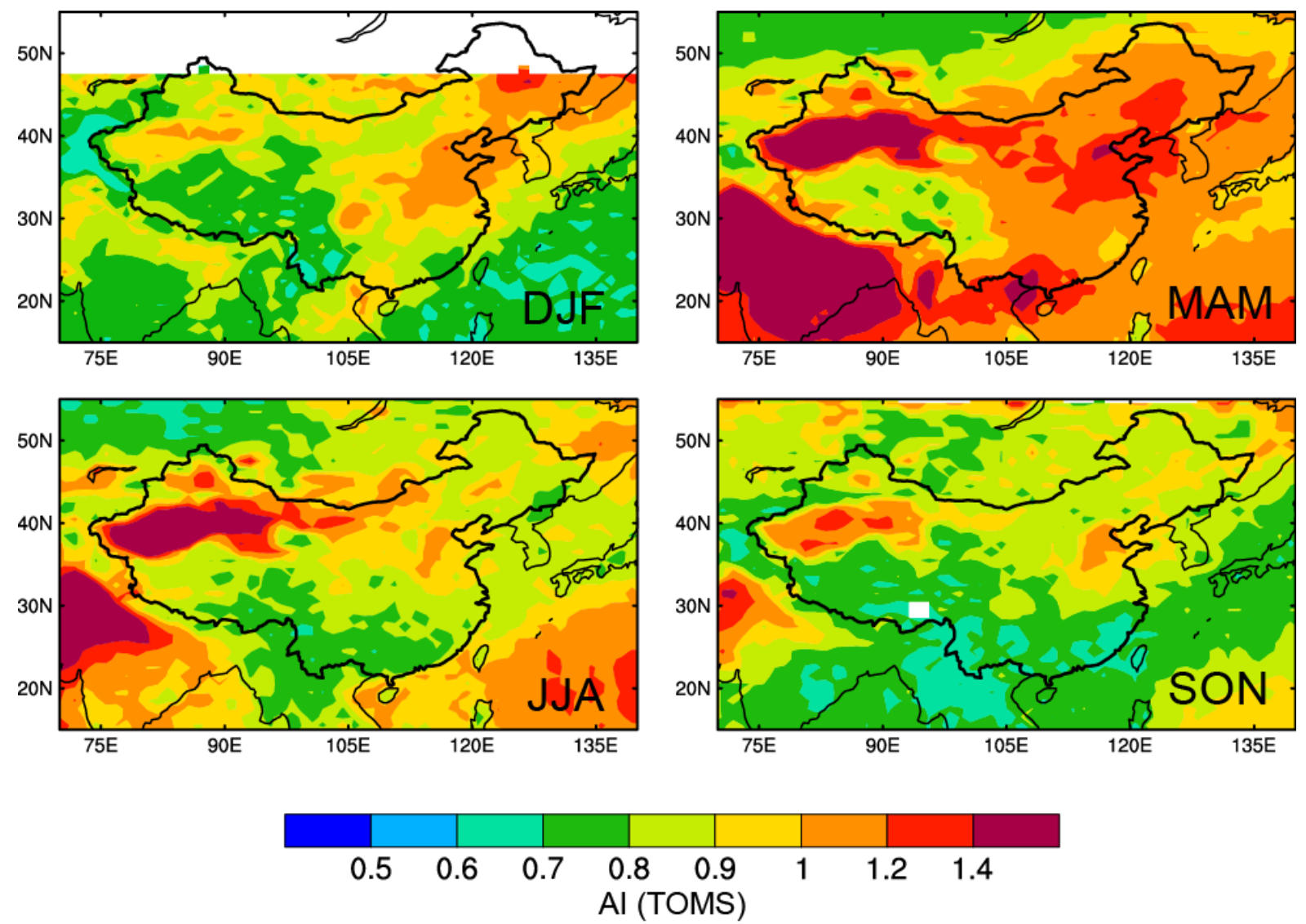

60 Figure S2. Spatial distribution of seasonal mean Aerosol Index (AI) derived from 61 Total Ozone Mapping Spectrometer (TOMS) measurements over years of 1997622004. 
DJF
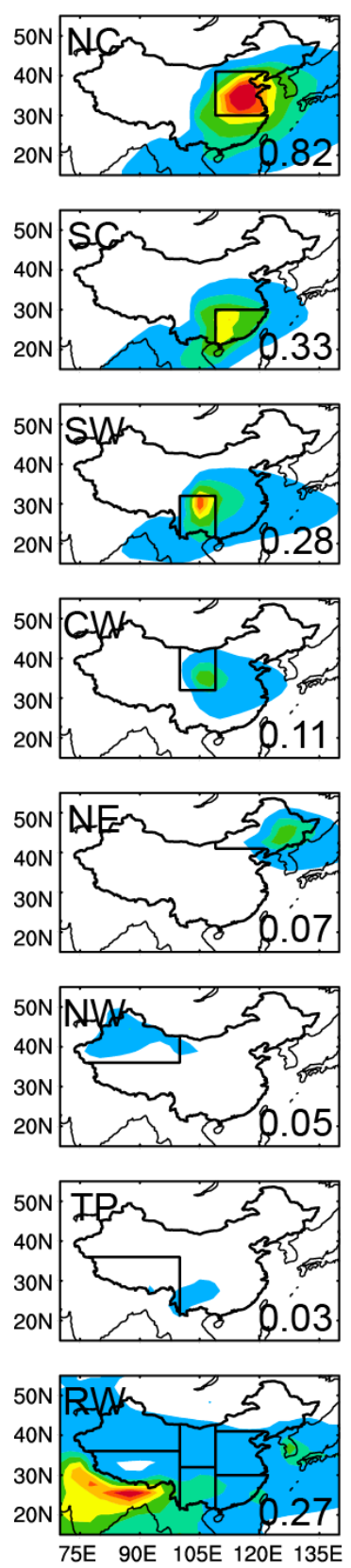

MAM
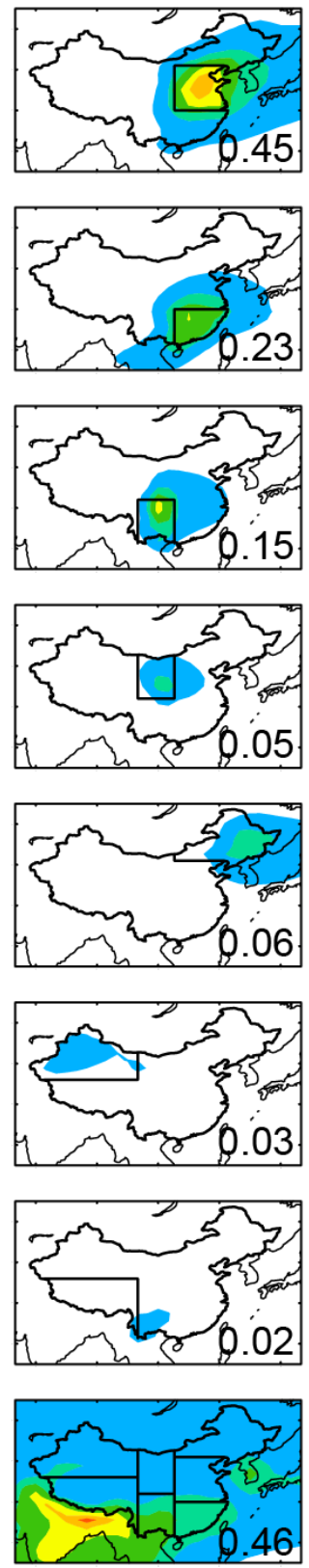

SON
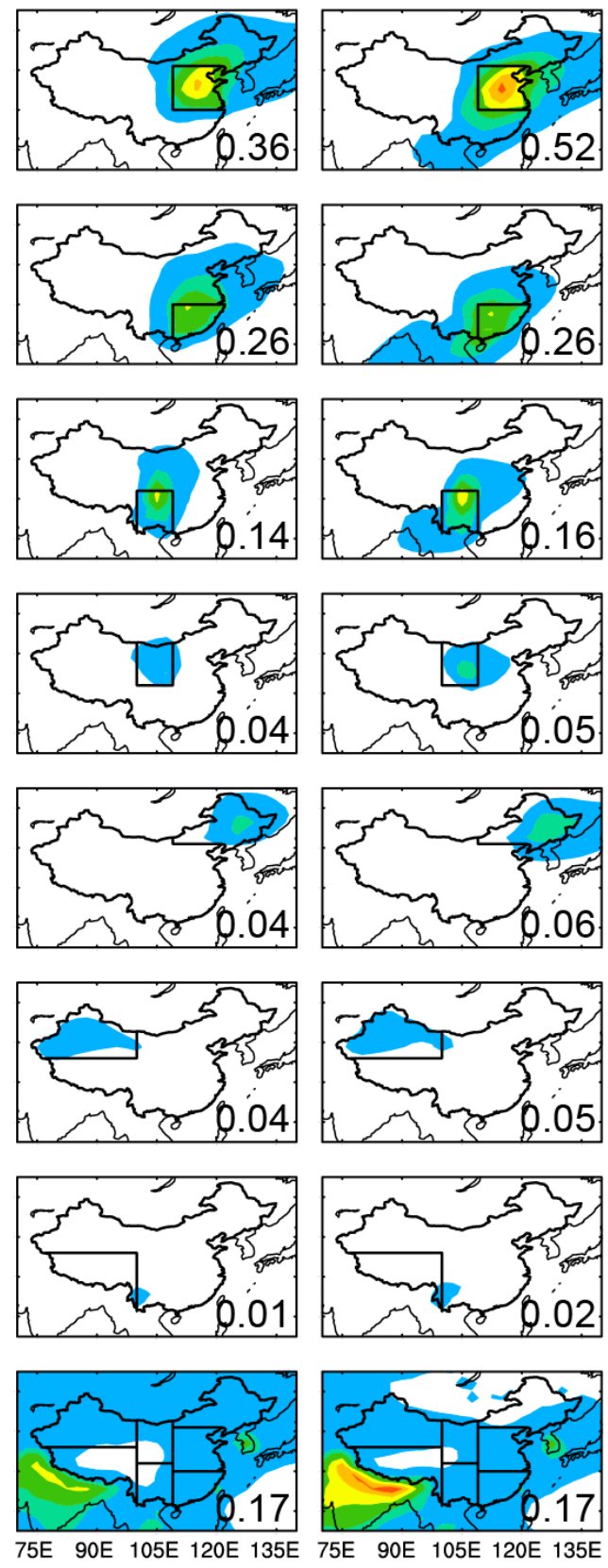

7

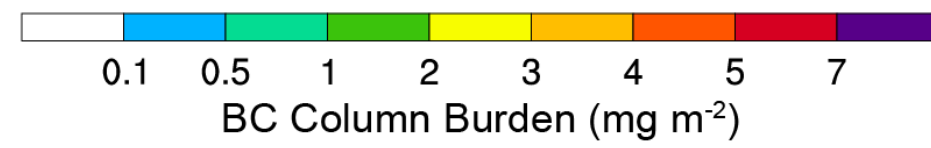

Figure S3. Spatial distribution of seasonal mean BC column burden $\left(\mathrm{mg} \mathrm{m}^{-2}\right)$ originating from the seven source regions in China (NC, SC, SW, CW, NE, NW, and TP), marked with black outlines, and sources outside China (RW). Regionally averaged BC in China contributed by individual source regions is shown at the bottom right of each panel. 
DJF
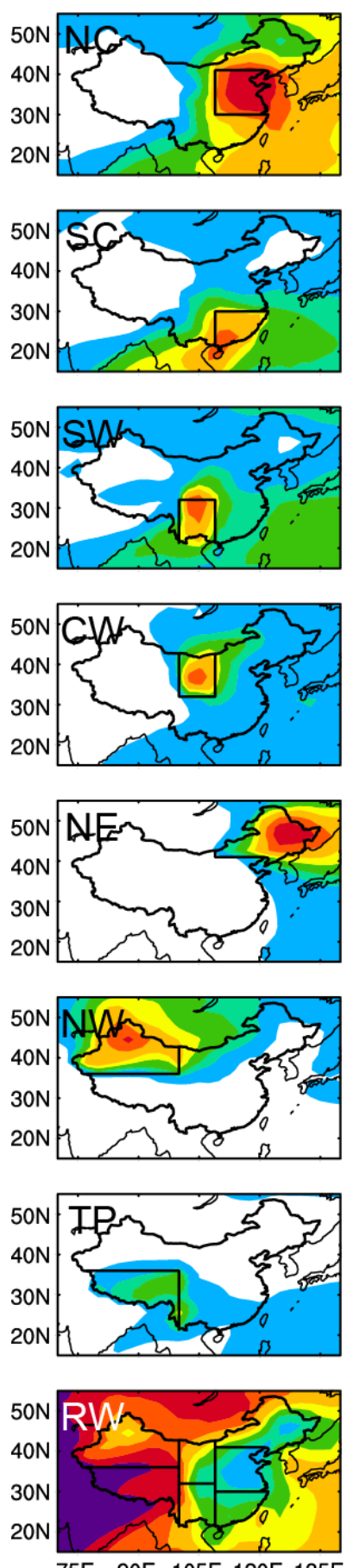

$75 E$ 90E 105E 120E 135E
MAM
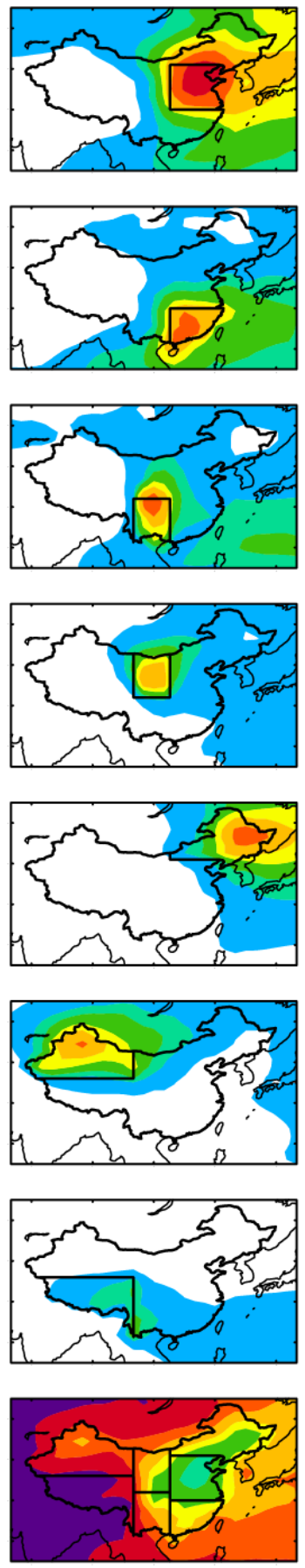

SON
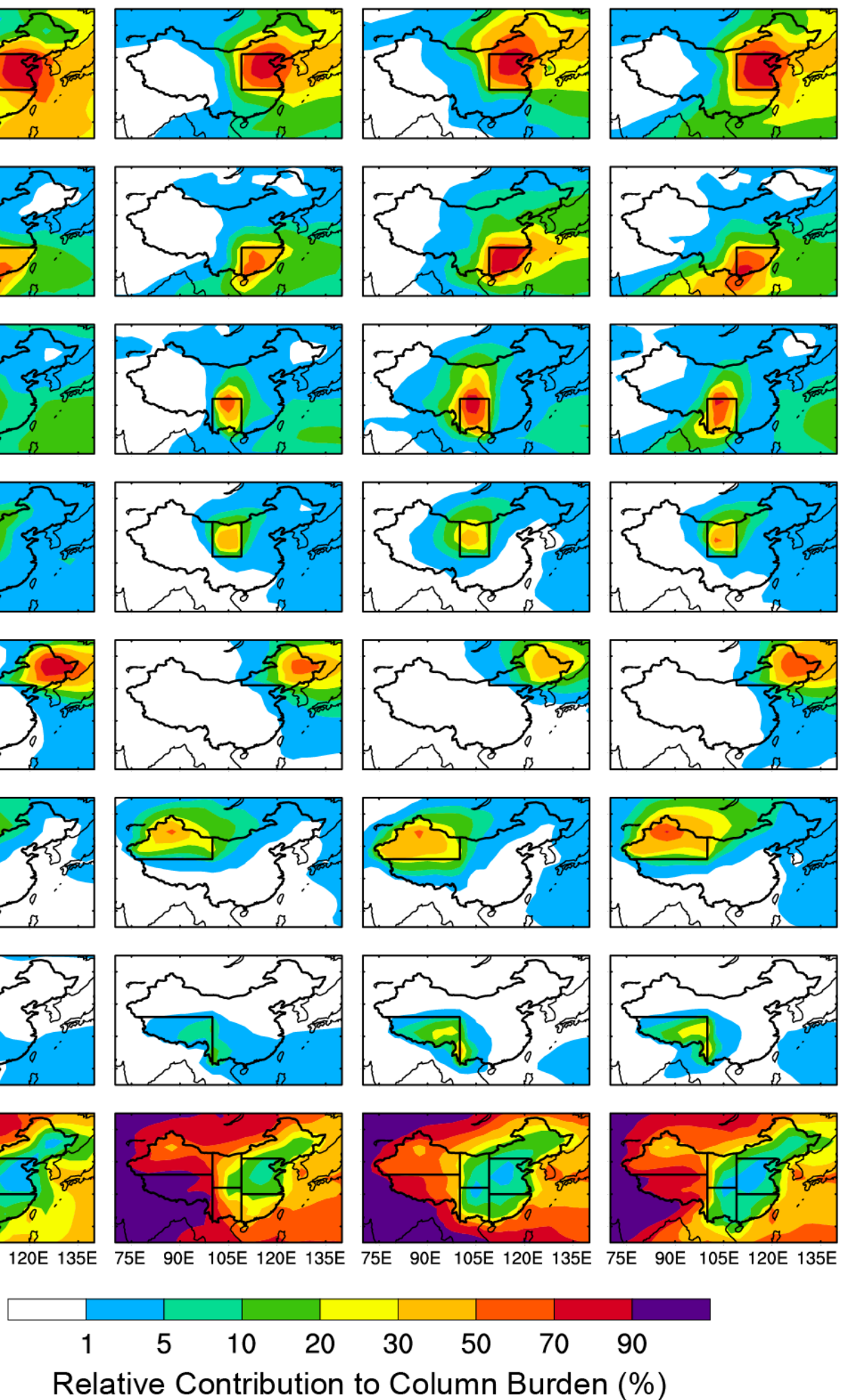

Relative Contribution to Column Burden (\%)

Figure S4. Spatial distribution of relative contributions (\%) to seasonal mean BC column burden from each of the tagged source regions. 


\section{Wind fields at $850 \mathrm{hPa}$}
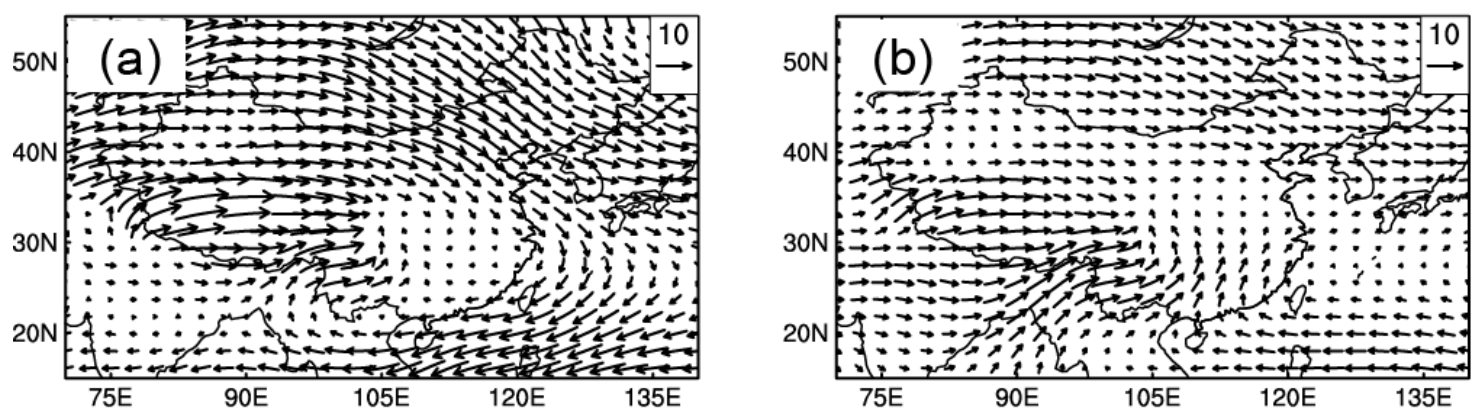

Figure S5. Simulated seasonal mean winds at $850 \mathrm{hPa}\left(\mathrm{m} \mathrm{s}^{-1}\right)$ in (a)
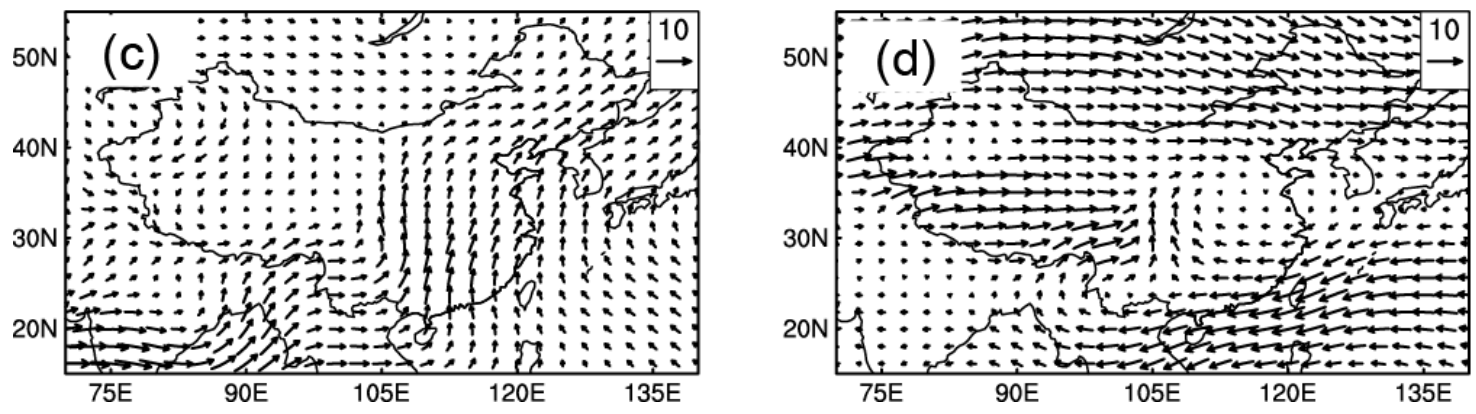

78

December-January-February (DJF), (b) March-April-May (MAM), (c) June-July-August (JJA), and (d) September-October-November (SON). 

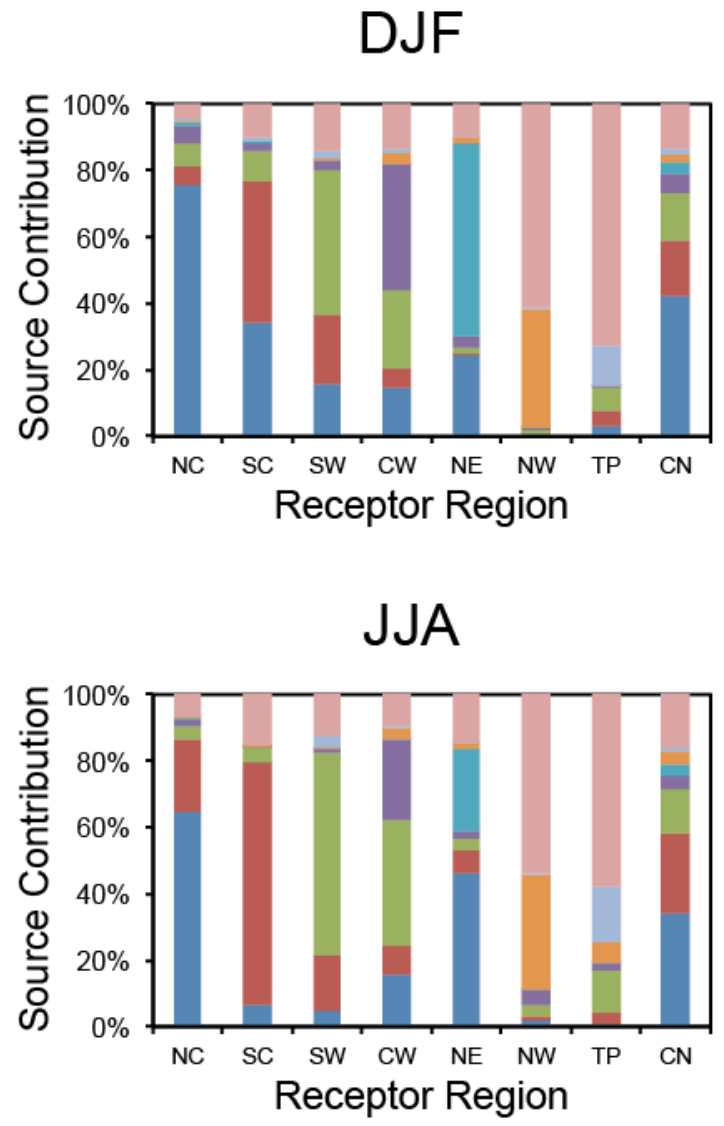

MAM

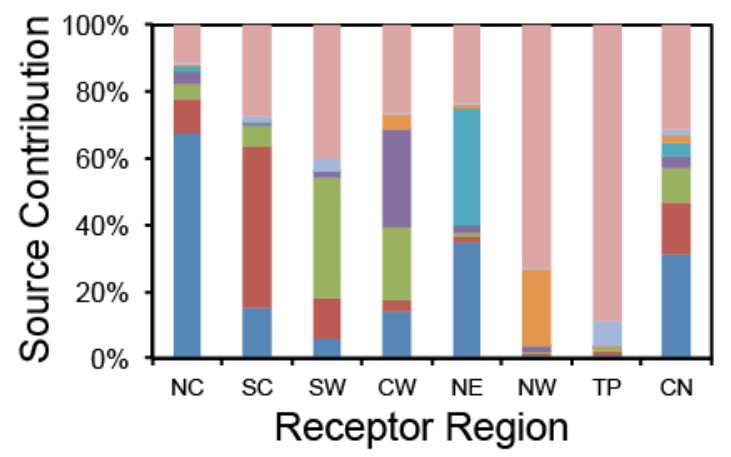

SON

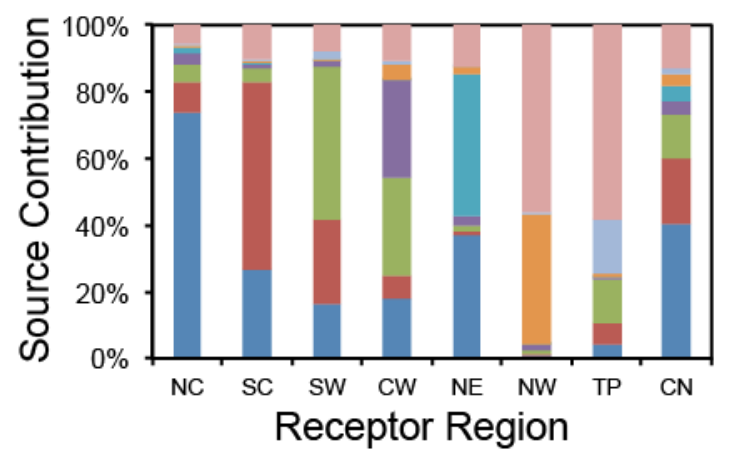

Source Region:

81 Figure S6. Relative contributions (\%) from the tagged source regions (denoted by 82 colors) to regional mean $\mathrm{BC}$ column burden over seven receptor regions in China 83 (NC, SC, SW, CW, NE, NW, and TP) and all of China (CN) in different seasons. The 84 receptor regions are marked on the horizontal axis in each panel. 

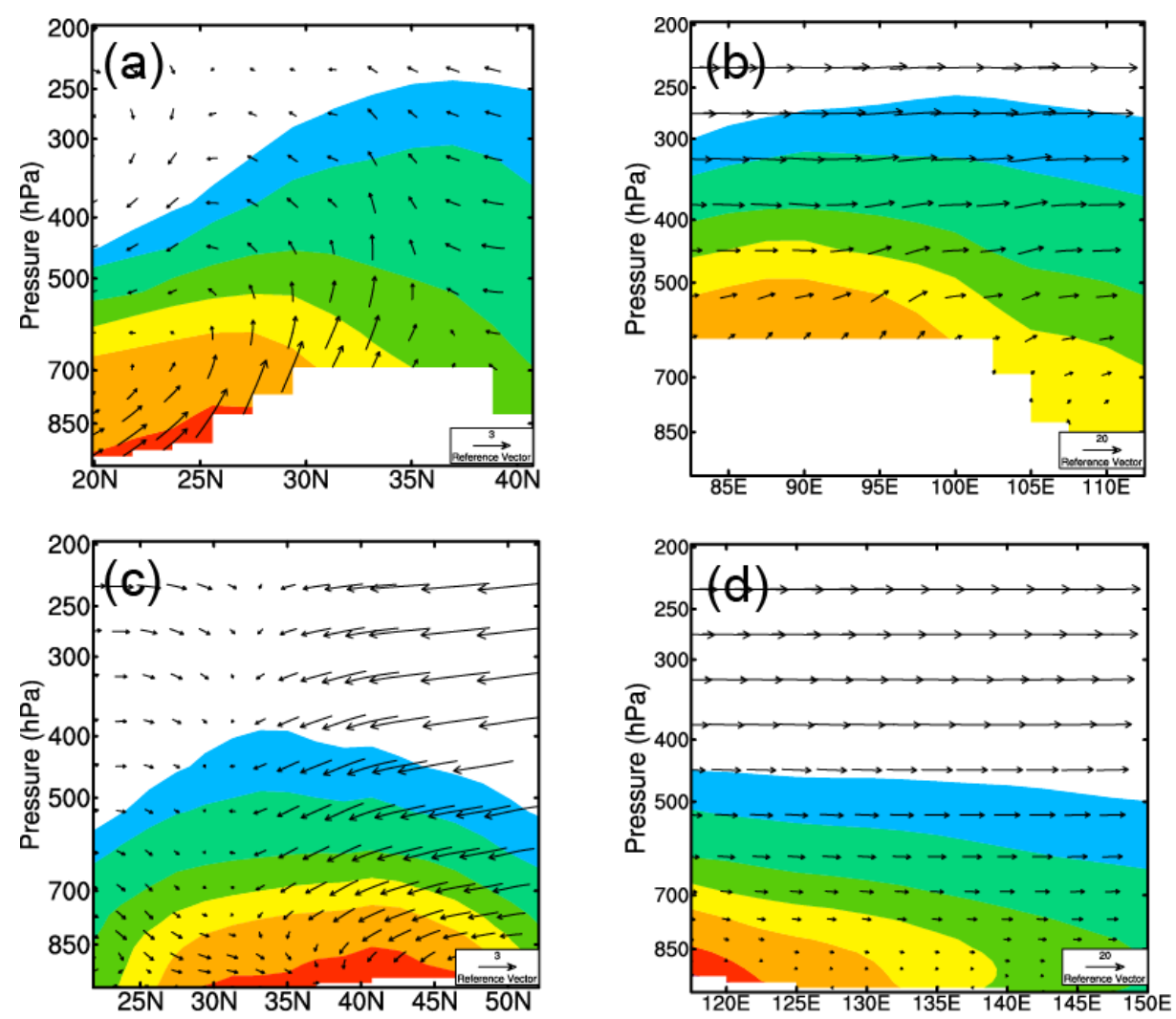

$$
0.010 .020 .05 \quad 0.1 \quad 0.2 \quad 0.5 \quad 1
$$

BC Conc. $\left(\mu \mathrm{g} \mathrm{m}^{-3}\right)$

Figure S7. Vertical distributions of $B C$ concentrations $\left(\mu \mathrm{g} \mathrm{m}^{-3}\right)$, originating from emissions outside China, averaged over (a) $75^{\circ}-120^{\circ} \mathrm{E}$ and (b) $25^{\circ}-35^{\circ} \mathrm{N}$, respectively, near the south boundary of China, and $\mathrm{BC}$ originating from total emissions in China averaged over (c) $120^{\circ}-135^{\circ} \mathrm{E}$ and (d) $20^{\circ}-50^{\circ} \mathrm{N}$, respectively, near the east boundary of China. 